\title{
Structure of Spinning Particle Suggested by Gravity, Supergravity and Low Energy String Theory
}

\author{
A. Burinskii \\ Gravity Research Group, NSI Russian Academy of Sciences, \\ B. Tulskaya 52, Moscow 113191, Russia, e-mail: grg@ibrae.ac.ru*
}

October, 1999

\begin{abstract}
The structure of spinning particle suggested by the rotating KerrNewman ( black hole ) solution, super-Kerr-Newman solution and the Kerr-Sen solution to low energy string theory is considered. Main peculiarities of the Kerr spinning particle are discussed: a vortex of twisting principal null congruence, singular ring and the Kerr source representing a rotating relativistic disk of the Compton size. A few stringy structures can be found in the real and complex Kerr geometry. Low-energy string theory predicts the existence of a heterotic string placed on the sharp boundary of this disk. The obtained recently supergeneralization of the Kerr-Newman solution suggests the existence of extra axial singular line and fermionic traveling waves concentrating near these singularities.

We discuss briefly a possibility of experimental test of these predictions.
\end{abstract}

\section{Introduction}

The Kerr solution is well known as a field of the rotating black hole. However, for the case of a large angular momentum $L,|a|=L / m \geq m$, all the horizons of the Kerr metric are absent, and the naked ring-like singularity is appeared. This naked singularity has many unpleasant manifestations and must be hidden inside a rotating disk-like source. The Kerr solution with $|a| \gg m$ displays some remarkable features indicating a relation to the structure of the spinning elementary particles.

${ }^{*}$ Talk at the International Workshop Spin'99, Prague, 5-11 September, 1999 
In the 1969 Carter [1] observed, that if three parameters of the Kerr - Newman solution are adopted to be $(\hbar=\mathrm{c}=1) \quad e^{2} \approx 1 / 137, \quad m \approx 10^{-22}, \quad a \approx$ $10^{22}, L=m a=1 / 2$, then one obtains a model for the four parameters of the electron: charge, mass, spin and magnetic moment, and the giromagnetic ratio is automatically the same as that of the Dirac electron. Israel [2] has introduced a disk-like source for the Kerr field, and it was shown by Hamity [3] that this source represents a rigid relativistic rotator. A model of "microgeon" with Kerr metric was suggested [- 1 and an analogy of this model with string models [5]. Then a model of the Kerr-Newman source in the form of oblate spheroid was suggested [7]. It was shown that material of the source must have very exotic properties: null energy density and negative pressure. An attempt to explain these properties on the basis of the volume Casimir effect was given in work [6]. The electromagnetic properties of the material are close to those of a superconductor [6, 7], that allows to consider singular ring of the Kerr source as a closed vortex string like the Nielsen-Olesen and Witten superconducting strings. Since 1992 black holes have paid attention of string theory. In 1992 the Kerr solution was generalized by Sen to low energy string theory [8]. It was shown that black holes can be considered as fundamental string states, and the point of view has appeared that some of black holes can be treated as elementary particles [9]. The obtained recently super-Kerr-Newman solution 10, 11] represents a natural combination of the Kerr spinning particle and superparticle models and predicts the existence of extra axial singularity and fermionic traveling waves on the Kerr-Newman background.

\section{Kerr singular ring}

The Kerr string-like singularity appears in the rotating BH solutions instead of the point-like singularity of the non-rotating BH. The simple solution possessing the Kerr singular ring was obtained by Appel in 1887 (!) [12]. It can be considered as a Newton or Coulomb analogue to the Kerr solution. When the point-like source of the Coulomb solution $f=1 / \tilde{r}=1 / \sqrt{\left(x-x_{o}\right)^{2}+\left(y-y_{o}\right)^{2}+\left(z-z_{o}\right)^{2}}$ is shifted to a complex point of space $\left(x_{o}, y_{o}, z_{o}\right) \rightarrow(0,0, i a)$, the Kerr singular ring arises on the real slice of space-time. The complex equation of singularity $\tilde{r}=0$ represents a ring as an intersection of plane and sphere. The complex radial distance $\tilde{r}$ can be expressed in the oblate spheroidal coordinates $r$ and $\theta: \tilde{r}=r+i a \cos \theta$. The Kerr singular ring is a branch line of the space on two sheets: "positive" one covered by $r \geq 0$, and "negative" one, an anti-world, covered by $r \leq 0$. The sheets are connected by disk $r=0$ spanned by singular ring. The physical fields change signs and directions on the "negative "sheet. Truncation of the negative sheet allows one to avoid the twosheetedness. In this case the fields will acquire a shock crossing the disk, and some material sources have to be spread on the disk surface to satisfy the field equations. The structure of electromagnetic field near the disk suggests then that the "nega- 
tive" sheet of space can be considered as a mirror image of the real world in the rotating superconducting mirror.

The source of Kerr-Newman solution, like the Appel solution, can be considered from complex point of view as a "particle" propagating along a complex world-line $x^{i}(\tau)$ [13, 14] parametrized by complex time $\tau$. The objects described by the complex world-lines occupy an intermediate position between particle and string. Like the string they form the two-dimensional surfaces or the world-sheets in the space-time. It was shown that the complex Kerr source may be considered as a complex hyperbolic string which requires an orbifoldlike structure of the world-sheet. It induces a related orbifold-like structure of the Kerr geometry 14] which is closely connected with the above mentioned twosheetedness.

\section{Kerr congruence and disk-like source}

Second remarkable peculiarity of the Kerr solution is the twisting principal null congruence (PNC) which can be considered as a vortex of null radiation. This vortex propagates via disk from negative sheet of space onto positive one forming a caustic at singular ring. PNC plays fundamental role in the structure of the Kerr geometry. The Kerr metric can be represented in the Kerr-Schild form $g_{i k}=\eta_{i k}+2 h k_{i} k_{k}$, where $\eta$ is metric of an auxiliary Minkowski space and $h$ is a scalar function. Vector field $k_{i}(x)$ is null, $k_{i} k^{i}=0$, and tangent to PNC. The Kerr PNC is geodesic and shear free [15]. Congruences with such properties are described by the Kerr theorem via complex function $Y(x)$ representing a projective spinor coordinate $Y(x)=\bar{\Psi}^{2} / \bar{\Psi}^{i}$. The null vector field $k_{i}(x)$ can be expressed in spinor form $k \sim \bar{\Psi} \sigma_{i} d x^{i} \Psi$.

The above complex representation of source allows one to obtain the Kerr congruence by a retarded-time construction [13, 14]. The complex light cone with the vertex at some point $x_{0}$ of the complex world line $\left(x_{i}-x_{0 i}\right)\left(x^{i}-x_{0}^{i}\right)=0$ can be split into two families of null planes: "left" and "right". In spinor form this splitting can be described as

$$
x_{i}=x_{0 i}+\Psi \sigma_{i} \tilde{\Psi}
$$

where "right" (or "left") null planes can be obtained keeping $\Psi$ constant and varying $\tilde{\Psi}$, or keeping $\tilde{\Psi}$ constant and varying $\Psi$. The rays of the twisting Kerr congruence arise as real slice of the "left" null planes of the complex light cones emanated from the complex world line [13, 14.

Replacement of the negative sheet by a disk-like source at surface $r=0$ allows one to avoid twosheetedness of the Kerr space. However, there is still a small region of causality violation on positive sheet of space. By the Löpez suggestion this region has to be also covered by source [7]. The minimal value of $r$ covering this region is 'classical radius' $r_{e}=\frac{e^{2}}{2 m}$. The resulting disk-like source has a thickness of order $r_{e}$ and its degree of oblateness is $\alpha^{-1} \approx 137$. 


\section{$4 \quad$ Stringy suggestions}

In 1974, in the frame of Einstein gravity the model of microgeon with the KerrNewman metric was considered [4], where singular ring was used as a waveguide for wave excitations. It was recognized soon [5] that singular ring represents in fact a string with traveling waves. Further, in dilaton gravity, the string solutions with traveling waves have paid considerable attention. The obtained by Sen generalization of the Kerr solution to low energy string theory with axion and dilaton [8] was analyzed in [16]. It was shown that, in spite of the strong deformation of metric by dilaton (leading to a change the type of metric from type D to type I), the Kerr PNC survives in the Kerr-Sen solution and retains the properties to be geodesic and shear free. It means that the Kerr theorem and the above complex representation are valid for the Kerr-Sen solution too. It has also been obtained that the field of the Kerr-Sen solution near the Kerr singular ring is similar to the field around a fundamental heterotic string that suggested stringy interpretation of the Kerr singular ring.

\section{Supergeneralization}

Description of spinning particle based only on the bosonic fields cannot be complete. On the other hand the fermionic models of spinning particles and superparticles based on Grassmann coordinates have paid considerable attention. In [10, 11] a natural way to combine the Kerr spinning particle and superparticle models was suggested leading to a non-trivial super-Kerr-Newman black hole solution.

The simplest consistent supergeneralization of Einstein gravity represents an unification of the gravitational field $g_{i k}$, with a spin $3 / 2$ Rarita-Schwinger field $\psi_{i}$. There exists the problem of triviality of supergravity solutions. Any exact solution of Einstein gravity is indeed a trivial solution of supergravity field equations with a zero field $\psi_{i}$. Starting from such a solution and using supertranslations, one can easily turn the gravity solution into a form containing the spin-3/2 field $\psi_{i}$. However, since this spin-3/2 field can be gauged away by reverse transformations such supersolutions have to be considered as trivial.

The hint how to avoid this triviality problem was given by complex representation of the Kerr geometry. One notes that from complex point of view the Schwarzschild and Kerr geometries are equivalent and connected by a trivial complex shift. The non-trivial twisting structure of the Kerr geometry arises as a result of the shifted real slice regarding the source [13, 14].

Similarly, it is possible to turn a trivial super black hole solution into a non-trivial if one finds an analogue to the real slice in superspace.

The trivial supershift can be represented as a replacement of the complex world line by a superworldline $X_{0}^{i}(\tau)=x_{0}^{i}(\tau)-i \theta \sigma^{i} \bar{\zeta}+i \zeta \sigma^{i} \bar{\theta}$, parametrized

by Grassmann coordinates $\zeta, \quad \bar{\zeta}$, or as a corresponding coordinate supershift 
$x^{\prime i}=x^{i}+i \theta \sigma^{i} \bar{\zeta}-i \zeta \sigma^{i} \bar{\theta} ; \quad \theta^{\prime}=\theta+\zeta, \quad \bar{\theta}^{\prime}=\bar{\theta}+\bar{\zeta}$.

Assuming that coordinates $x^{i}$ before the supershift are the usual c-number coordinates one sees that coordinates acquire nilpotent Grassmann contributions after supertranslations. Therefore, there appears a natural splitting of the space-time coordinates on the c-number 'body'-part and a nilpotent part - the so called 'soul'. The 'body' subspace of superspace, or B-slice, is a submanifold where the nilpotent part is equal to zero, and it is a natural analogue to the real slice in complex case.

Reproducing the real slice procedure of the Kerr geometry in superspace one obtains the condition of proportionality of the commuting spinors $\bar{\Psi}(x)$ determining the PNC of the Kerr geometry and anticommuting spinors $\bar{\theta}$ and $\bar{\zeta}$, As a consequence of the B-slice and superlightcone constraints one obtains a submanifold of superspace $\theta=\theta(x), \quad \bar{\theta}=\bar{\theta}(x)$. The initial supergauge freedom is lost now, and there appears a non-linear realization of broken supersymmetry introduced by Volkov and Akulov [17, 18] and considered in $\mathrm{N}=1$ supergravity by Deser and Zumino [19. It is assumed that this construction is similar to the Higgs mechanism of the usual gauge theories, and $\zeta^{\alpha}(x), \quad \bar{\zeta}^{\dot{\alpha}}(x)$ represent Goldstone fermion which can be eaten by appropriate local supertransformation with a corresponding redefinition of the tetrad and the spin-3/2 field $\psi_{i}$.

However, the complex character of supertranslations demands to extend this scheme to $\mathrm{N}=2$ supergravity.

In this way the self-consistent super-Kerr-Newman solutions to broken $\mathrm{N}=2$ supergravity coupled to Goldstone fermion field was obtained [11]. The solution describes the massless Dirac wave field propagating on the Kerr-Newman background along the Kerr congruence. Besides the Kerr singular ring solution contains an extra axial singularity and traveling waves propagating along the ring-like and axial singularity.

The 'axial' singularity represents a half-infinite line threading the Kerr singular ring and passing to 'negative' sheet of the Kerr geometry. The position and character of axial singularity depend on the index $n$ of elementary excitation. The case $n=-1 / 2$ is exclusive: there are two 'decreasing' singularities which are situated symmetrically at $\theta=0$ and $\theta=\pi$.

\section{Problem of hard core}

The obtained supergeneralization is based on the massless Goldstone field. At present stage of investigation our knowledge regarding the origin of the Goldstone fermion is very incompleted. Analyzing the Wess-Zumino model of superQED and some other schemes of spontaneously broken supersymmetry [18], one sees that it can leads to massless Goldstone fermions, at least in the region of massless fields out of the $\mathrm{BH}$ horizons.

However, for the known parameters of spinning particles, the angular momentum is very high, regarding the mass parameter, and the black hole horizons 
disappear. The resulting object is "neither black and nor hole", and the considered above disk-like 'hard core' region is naked. Structure of this region represents a very important and extremely complicated problem. Among the possible field models for description this region could be mentioned the LandauGinzburg model, super-QED, non-abelian gauge models, Seiberg-Witten theory, as well as the recent ideas on the confinement caused by extra dimensions in the bulk/boundary ( AdS/CFT correspondence) models 21]. Apparently, this problem is very far from resolution at present, and one of the most difficult

points can be the concordance of the field model with the rotating disk-like bag of the Kerr geometry.

\section{Suggestions to experimental test}

The predicted comparative big size of the disk-like bag looks as a serious contradiction to the traditional point of view on the structureless, point-like electron. However, the suggested by QED virtual photons surrounding electron in the region of Compton size, zitterbewegung and the vacuum zero-point fluctuations, spreading the position of electron, can be treated as some indirect evidences for the existence of an geometrical structure in the Compton region. At least, one can assume that region of virtual photons has a tendency to be very ordered with formation of the Kerr congruence and the ring-like singularity.

The modern progress in the formation of polarized beams of spinning particles suggests the possible methods for experimental test of the main predicted feature of the Kerr spinning particle - its highly oblated form. In particular, it could be the method proposed in [20] based on estimation of the cross section differences between transversely and longitudinally polarized states in protonproton collisions. One proposes that similar experiment could be more effective for the electron-electron collisions. Another possible way of experimental test could be the analysis of the diffraction of photons on the polarized electrons.

Apparently, the strong influence of vacuum fluctuations will not allow one to observe the predicted very high oblateness of electrons. Nevertheless, one expects that an essential effect should be observed if the Kerr source model reflects the reality.

\section{References}

[1] Carter B., Phys. Rev. 174(1968) 1559;

[2] Israel W., Phys. Rev. D2 (1970) 641;

[3] Hamity V. Phys. Lett. A 56(1976)77;

[4] Burinskii A., Sov. Phys. JETP 39(1974) 193; 
[5] Ivanenko D. and Burinskii A., Izv. VUZ Fiz.5(1975)135; Sov. Phys. J. (USA).,nr.7 (1978)113;

[6] Burinskii A. Phys. Lett. B 216(1989)123;

[7] López C.A., Phys. Rev. D30 (1984) 313;

[8] Sen A., Phys.Rev.Lett., 69(1992)1006;

[9] Dabholkar A., Gauntlett J., Harvey J. and Waldram D., Nucl. Phys. B 474(1996) 85;

Sen A. Modern Phys. Lett. A 10(1995)2081; Nucl.Phys B46 (Proc.Suppl)(1996)198;

[10] Burinskii A., Phys.Rev.D 57(1998)2392, hep-th/9704102;

[11] Burinskii A. Class.Quant. Grav. 16(1999)(Oct-Nov to appear) hepth/9903032;

[12] E.T. Whittacker and G.N. Watson, A Course of Modern Analysis, Cambrige Univ. Press London/New York,p.400, 1969 .

[13] Burinskii A., Kerr R.P. and Perjes Z., 1995, Nonstationary Kerr Congruences, gr-qc/9501012;

[14] Burinskii A., String - like Structures in Complex Kerr Geometry, Proc. of the Fourth Hungarian Relativity Workshop Edited by R.P. Kerr and Z. Perjes, Academiai Kiado, Budapest 1994, gr-qc/9303003;

Burinskii A., Phys.Lett. A 185(1994)441; Burinskii A. Espec. Space Explorations, 9 (C2)(1995) 60, Moscow, Belka, hep-th/9503094;

[15] Debney G.C., Kerr R.P., Schild A., J.Math.Phys.,10(1969)1842;

[16] Burinskii A., Phys.Rev.D 52(1995)5826, hep-th/9504139;

[17] Volkov D.V. and Akulov V.P. Pis'ma Zh. Eksp.Teor.Fiz. 16621(1972);

[18] Wess J. and Bagger J. Supersymmetry and Supergravity, Princeton, New Jersey 1983;

[19] Deser S. and Zumino B. Phys. Rev. Lett., 38(1977) 1433;

[20] Musulmanbekov G. Total Cross Section Differences Between Transversely and Longitudinally Polarized States in Proton-Proton Collisions. In: Proc. of VII Workshop on High Energy Physics SPIN'97, July 1997, Dubna, Russia, Dubna 1997, p.165. 
[21] E. Witten, Adv. Theor. Math. Phys. 2 (1998) 253, hep-th/9802150; J. Maldacena, Adv. Theor. Math. Phys. 2 (1998) 231, hep-th/9711200; I.R. Klebanov and A.A. Tseytlin, hep-th/9812089; J.A. Minahan, JHEP bf 9901(1999)020, hep-th/9811156, hep-th/9902074: A.Kehagias and K. Sfetsos, Phys.Lett.B454(1999)270, hep-th/9902125;iibid.B456(1999)22, hepth/9903109; H.Liu and A.A. Tseytlin, hep-th/9903091; R. de Mello Koch, A. Paulin-Campbell and J. P. Rodrigues, hep-th/9903029; S. Nojiri and S.D. Odintsov, hep-th/9904036, hep-th/9906216; A. Burinskii, hepth/9908198 